\title{
Seropositivity and risk factors of Toxocara canis infection in adult asthmatic patients
}

\author{
Rouhani-Rankouhi, S.Z. ${ }^{1}$, Kow, K.S. ${ }^{2}$, Liam, C.K. ${ }^{2}$ and Lau, Y.L. ${ }^{1 *}$ \\ ${ }^{1}$ Department of Parasitology, Faculty of Medicine, University of Malaya, 50603 Kuala Lumpur, Malaysia \\ ${ }^{2}$ Department of Medicine, Faculty of Medicine, University of Malaya, 50603 Kuala Lumpur, Malaysia \\ *Corresponding author e-mail: lauyeeling@um.edu.my \\ Received 17 February 2020; received in revised form 14 May 2020; accepted 17 May 2020
}

\begin{abstract}
This cross-sectional study involving 86 adult asthmatic patients aimed to determine the relationship between Toxocara seropositivity and severity of asthma in adult asthmatics and investigate the risk factors for Toxocara infection. In all cases, T. canis IgG level was measured using an anti-Toxocara IgG enzyme-linked immunosorbent assay kit. Total serum IgE and eosinophil count were also determined. The anti-Toxocara IgG seropositivity was $68.6 \%$ among asthmatic patients. There were no statistically significant associations between Toxocara seroprevalence and other risk factors, clinical symptoms of asthma and high level of total serum IgE and eosinophilia. Pet ownership could be an important risk factor for Toxocariasis. Having a pet at home and wheezing were significantly associated with Toxocara seropositivity in adult asthmatic patients.
\end{abstract}

\section{INTRODUCTION}

Toxocariasis is a human parasitic disease caused by Toxocara canis and Toxocara cati, roundworms of dogs and cats respectively. Toxocariasis is common in developing countries, especially in tropical regions and among poor population. Dogs are definitive hosts and can be infected by T. canis through ingestion of embryonated eggs or transmammary or trans-placental transmission of larvae. Human can be infected via ingestion of embryonated T. canis eggs as well. The major means of transmission of infection in human is ingestion of embryonated eggs excreted by dogs' feces found in contaminated soil or uncooked meat or unwashed vegetables or fruits, or through accidental consumption of raw/undercook paratenic host meat containing infective larvae (Yoshida et al., 2016). Although human is a paratenic host for T. canis, the parasite cannot be developed into an adult worm. Few larvae are required to cause disease in humans. Most infections by T. canis is asymptomatic such as covert-toxocariasis or (CT) and the morbidity of this syndrome depends on the parasitic burden and host immune system bias. Immunological detection assays for toxocariasis detection is based on native or recombinant TES antigens or either glycan antigens or deglycosylated TES antigens and antibodies like total IgG, IgG subclasses or IgM (Ma et al., 2014).

Epidemiological studies suggest that infections with Toxocara can cause allergic reaction such as asthma with symptoms like wheezing, coughs, mucus hyper-secretion and bronchial hyper-reactivity (Pinelli $e t$ $a l ., 2012)$. Hygiene hypothesis suggest that helminth infections such as Ascaris lumbricoides, Schistosoma mansoni and Trichuris trichiura can protect human body from allergic diseases, whereas Toxocara can develop this immunopathology in children in particular.

Although few studies suggested no corelation between Toxocara infection and asthma, no studies have been reported on an inversion association. Hygiene hypothesis 
suggested that because of lack of infection in children there is weaker Th1 response which allows increasing of Th2 response toward environmental allergens. Interaction between helminth infection and allergy involved Treg cells (Pinelli et al., 2012).

Toxocara infection is associated with a polarized $\mathrm{CD} 4^{+} \mathrm{Th} 2$ response with high level of IgE and eosinophilia which are mediated by HLA class II molecules. There is association between HLA class II and pathological severity. Besides, it has been suggested that Foxp3 $3^{+} \mathrm{CD} 4^{+} \mathrm{CD} 25^{+}-$ expressing $\mathrm{T}$ regulatory (Treg) cells can regulate the immunopathology of granulomas in experimental toxocaral granulomatous hepatitis and can increase the level of TGF- $\beta 1$ expression level which is important for local survival and function of Treg during invasion of T. canis in intestine, live, muscle and brain (Fan et al., 2013).

Toxocariasis is associated with elevated levels of specific IgE against aeroallergens ( $\mathrm{sIgE})$, serum total $\mathrm{IgE}$, eosinophilia, increased skin sensitivity to aeroallergens, atopic asthma and decreased lung function. Toxocara can cause allergy reactions by inducing Th2 response. Glycan antigens, which are located on glycoproteins of Toxocara larvae, have an important role in induction of this lymphocyte that consequently increases the production of IL- 4 and switches B cells for IgE synthesis. Further, Th2-type lymphocyte increases production of IL-5, which increases the total numbers of eosinophils, macrophages and mast cells. Eosinophilia causes smooth muscle layers to thicken and consequently narrows down the airways. Besides, eosinophils can produce free radicals that damages trachea and causes pulmonary inflammation. Asthma is the most wide spread pulmonary disease in the world and its prevalence is $0-30 \%$ in children and around $10 \%$ in adults. The clinical symptoms of asthma include wheezing, cough, mucus hyper-secretion and bronchial hyperreactivity (Mosayebi et al., 2016; Pinelli et al., 2012; Qualizza et al., 2009). Atopy is the most important risk factor of asthma because about half of asthma cases are atopic, while epidemiological studies suggest that Toxocara plays a major role in pathogenesis of atopy (Pinelli et al., 2012). Experimental studies suggested the association and corelation of toxocariasis and development of allergic disease such as asthma (Li et al., 2014). It has been identified by Li et al. (2014) that there is association between toxocariasis and lung function. Toxocariasis is a common helminth infection, therefore detection and estimation of Toxocara infection and asthma is necessary ( $\mathrm{Li}$ et al., 2014). The aim of this study was to determine the relationship between Toxocara seropositivity in adult asthmatic patients, and to investigate the risk factors of Toxocara infection.

\section{MATERIAL AND METHODS}

\section{Study population}

A cohort of 86 adult asthmatic patients aged between 19 and 92 years participated in this study between August 2016 and February 2017 at University Malaya Medical Centre. The study protocol was accepted by Ethics Committee of Faculty of Medicine, University of Malaya (MEDIC.NO: 201612042). Informed consent forms were filled out by all patients. Risk factors shown in Table 1 were used, based on the published papers and ISSAC questionnaire.

\section{Measurement of total IgE ELISA kit} using adult asthmatic serum samples

Eighty-six asthmatic adults' sera were used in a commercial total Toxocara IgE Enzyme Immunoassay Test Kit (BioCheck, Inc, CA, USA). Twenty microliters of standard specimens and controls were dispensed into appropriate wells in ELISA microtiter plate. One hundred microliter of blocking buffer were added to each well. The plate was thoroughly mixed on a plate shaker for 30 seconds. The plate was incubated at room temperature $\left(18\right.$ to $\left.25^{\circ} \mathrm{C}\right)$ for 30 minutes. The plate was washed and rinsed 5 times in distilled or deionized water. After the last rinse, the wells were slapped out on a clean absorbent towel to remove excess wash buffer. Hundred and fifty microliters of 
enzyme conjugate reagent were added to all the wells, gently mixed for 10 seconds. The plate was incubated at room temperature for 30 minutes. The plate was washed and rinsed 5 times in distilled or deionized water. After the last rinse, the wells were slapped out on a clean absorbent towel to remove excess water. One hundred microliters of TMB reagent were added to each well and gently mixed for 10 seconds. The plate was incubated at room temperature for 20 minutes. One hundred microliters of reaction stopping solution were added to each well and were gently mixed for 30 seconds. The optical density (OD) was read at $450 \mathrm{~nm}$ with microtiter plate reader within 15 minutes.

\section{Measurement of Toxocara IgG ELISA using adult asthmatic serum samples}

Eighty-six adult asthmatic patients' sera were used in a Toxocara IgG Enzyme Immunoassay Test Kit (AccuDiag ${ }^{\mathrm{TM}}$ Toxocara IgG ELISA Kit; CA, USA). Patients' sera were diluted (1:64 dilution). One hundred microliters of negative control, positive control and diluted sera were added to the remaining wells. The ELISA plate was incubated at room temperature for 10 minutes. The wells were washed and rinsed three times using the wash buffer. After the last rinse, the wells were slapped out on a clean absorbent towel to remove excess wash buffer. One hundred microliters of enzyme conjugate were added to each well. The ELISA plate was incubated at room temperature for 5 minutes and then washed and rinsed three times. After the last rinse, the wells were slapped out on a clean absorbent towel to remove excess wash buffer. One hundred microliters of chromogen (TMB) were added. The ELISA plate was incubated at room temperature for 5 minutes. One hundred microliters of reaction stopping solution were added to each well. The ELISA plate was tapped gently on the side of the strip holder with index finger for approximately 15 seconds to mix wells. The absorbance of samples at wavelength of 450 nm was measured using a microplate reader. Absorbance read greater than, or equal to
0.2 OD units, was positive, while absorbance read less than 0.2 OD units was negative. A positive OD reading indicates that the patient may be infected by Toxocara.

\section{Eosinophil count}

Eosinophil count was performed by a laboratory technician at University Malaya Medical Center, Kuala Lumpur.

\section{Statistical analysis}

Statistical analysis in this study was performed using SPSS version 19. Chi-square test was conducted to conform the link between Anti Toxocara IgG and other variables. The level of significance selected was $p<0,05$.

\section{RESULTS}

The (mean+SD) of age for patients was $(64.19+15.107)$. Table 1 shows the distribution of study variables among study patients and association of these variables with Toxocara seroprevalence. Based on this study, there was a statistically significant association between Toxocara seroprevalence (Anti Toxocara IgG seropositivity) and pet ownership as well as having wheezing symptom in the past 12 months $(p<0.05)$. There was no statistical association between Toxocara seroprevalence and other study variables and outcomes among the patients under study. Besides, there was no association between Toxocara seroprevalence and increasing level of total IgE or eosinophilia $(p>0.05)$.

\section{DISCUSSION}

The possible role of $T$. canis in asthma is not clear. Respiratory changes occur in VLM and CT because of larval migration. According to Buijs et al. (1994), the antiToxocara antibodies in asthmatic children were higher than those in healthy children. Moreover, the seropositivity of Toxocara was higher among children aged between 11 and 15, compared to children aged between 2 


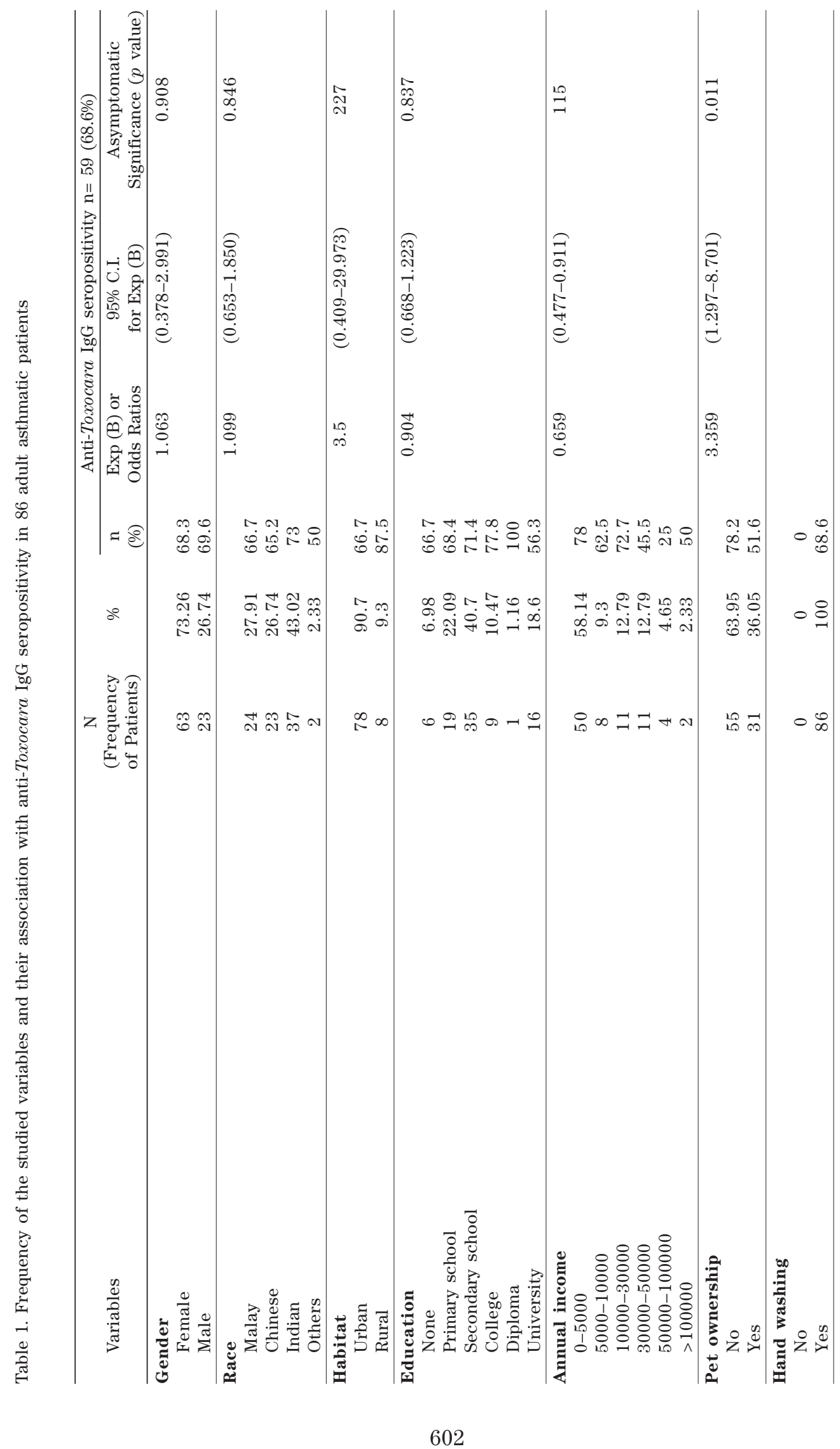




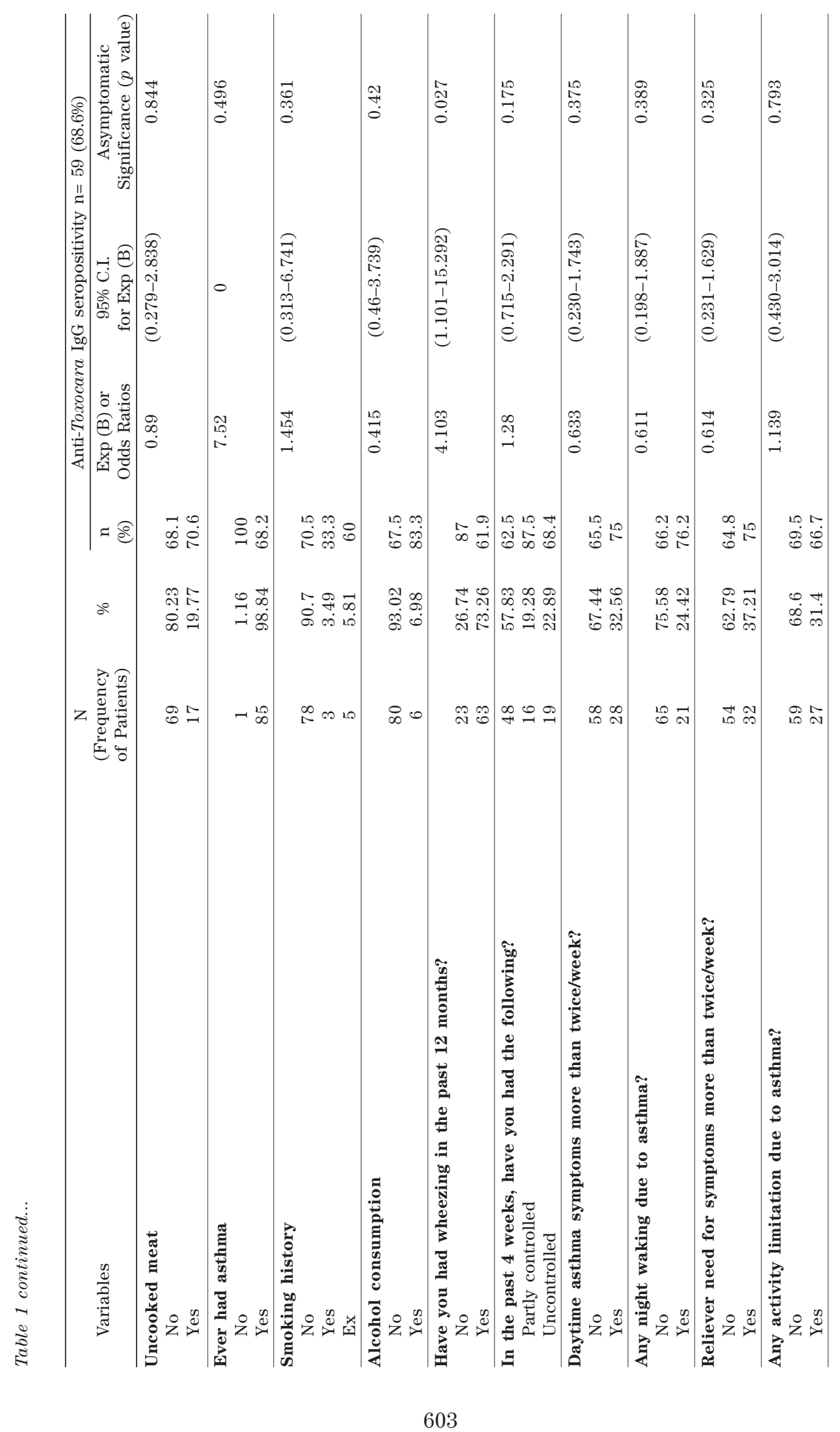




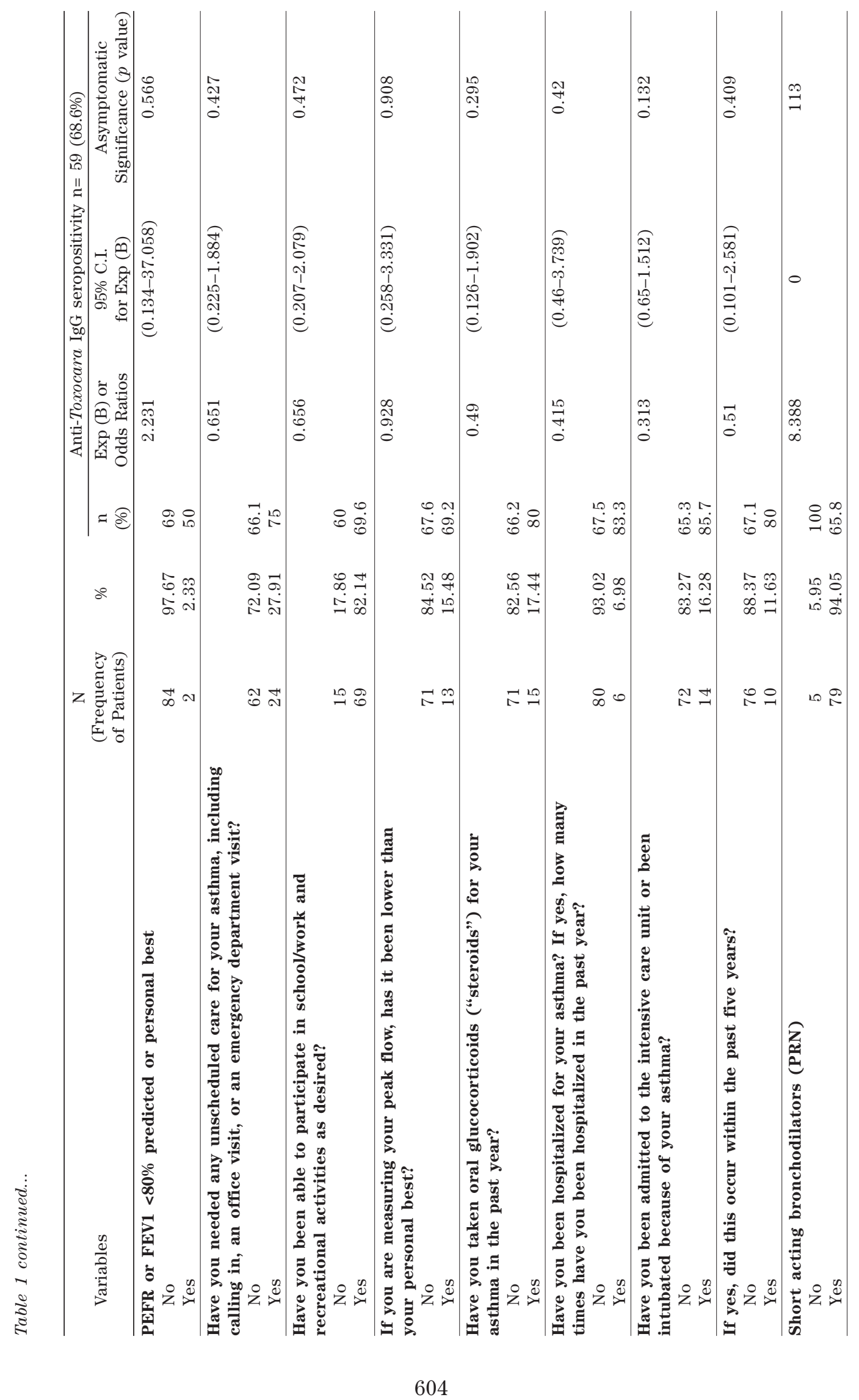




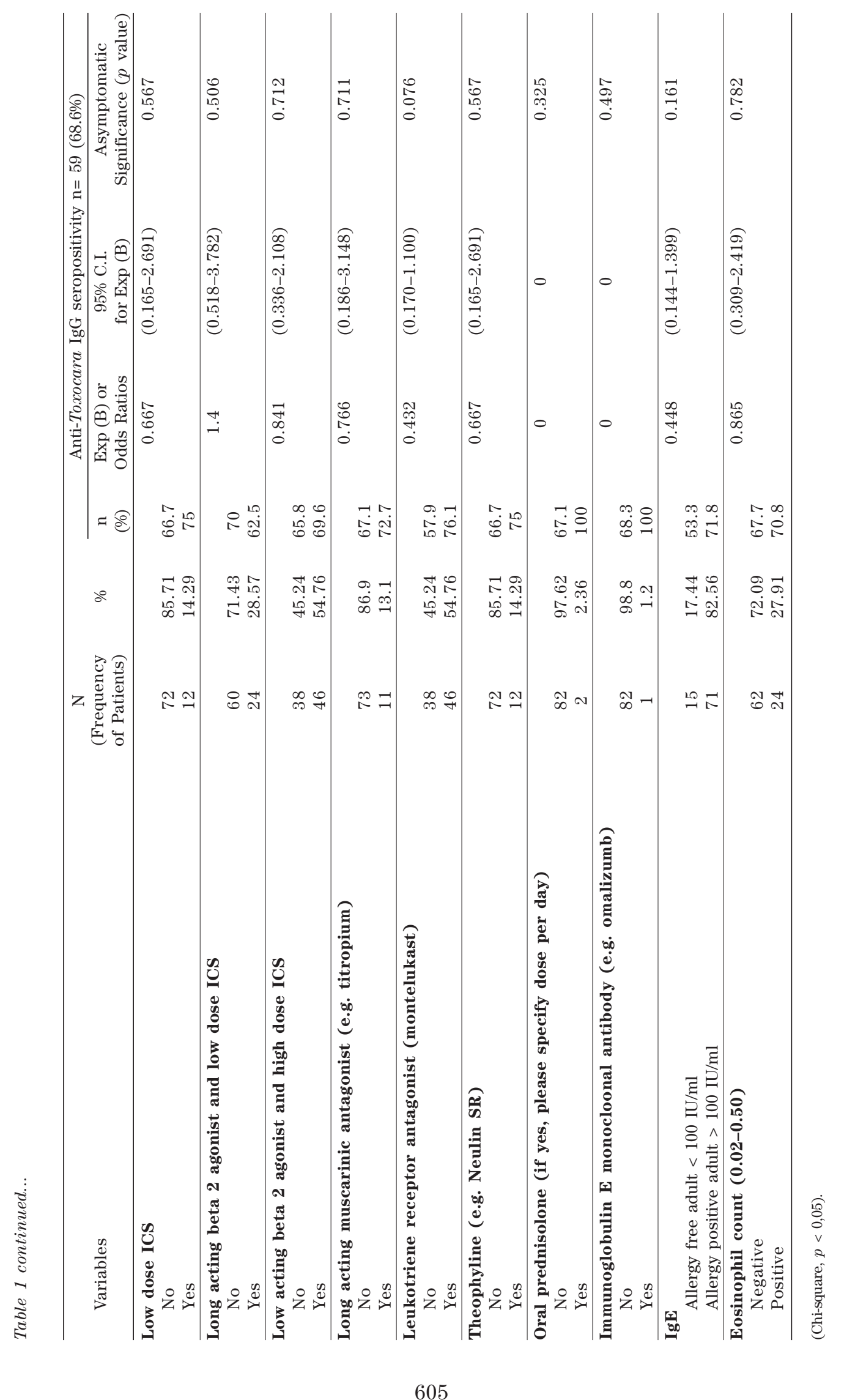


and 10. Fernando et al. (2009) indicated that $29 \%$ of children with bronchial asthma showed Toxocara seropositive manifestation. High eosinophilia was detected in $86 \%$ of these asthmatic children (Fernando et al., 2009). According to Mendonça et al. (2012), the prevalence of Toxocara in children from Salvador area in Brazil was found to be $47 \%$. There are few studies and reports indicating the relationship between asthma and toxocariasis among adults. Feldman et al. (1992) reported a high level of anti-Toxocara IgG in a 48-year-old asthmatic patient with a hypereosinophilic syndrome. According to Buijs et al. (1995), there was a marginal relation between eczema and Toxocara seropositivity. Ehrhard et al. (1979) observed transient rash, urticaria and hypodermic nodules in $23 \%$ of seropositive children and $29 \%$ of seropositive adults.

The rate of toxocariasis across Malaysia has been indicated to be around 20\%. This study focused on adult asthmatic patients, differ to previous studies conducted in Malaysia (Hakim et al., 1997) and in Brazil (Mendonça et al., 2012) while they studied the relationship between asthma and toxocariasis in children. Since biopsy, as the gold standard diagnostical test of toxocariasis, is laborious and time-consuming; thus, serological diagnosis is a more reliable test for detecting Toxocara. In the current study, 86 serum samples of adult asthmatic patients were obtained from UMMC hospital. Toxocara IgG ELISA was done to confirm toxocariasis. Out of 86 patients, 59 (68.60\%) were positive with toxocariasis, and 27 (31.39\%) were negative. We found no association between Toxocara infection and major immunological reactions such as marked eosinophila and IgE response. In this study, the risk factors that include age, gender, habitat, education level, pet ownership, washing hands before meals, raw meat consumption, family history of asthma disease and other variables, have been investigated based on previously published papers as well as ISSAC questionnaire. Similar to Sharghi et al. (2001), our results did not indicate any links between asthma and toxocariasis. We found that $36.05 \%$ of asthmatic patients had pets. This result is lower in percentage when compared to the study carried out by Mendonça et al. (2013), where $66.6 \%$ of the contact with puppies and $60 \%$ of the contact with adult dogs were reported positive. Based on the statistical analyses conducted by SPSS version 19, we found a significant relatiomship between Toxocara seroprevalence and pet ownership, as well as the pathogenesis of wheezing in the past 12 months. No relations were detected between Toxocara seroprevalence and other variables among adult asthmatic patients. One of the limitation of our study was lack of control in our research. Besides, Toxocara-specific IgE level were not tested in our study since we had only Total-IgE test.

\section{CONCLUSION}

In conclusion, this study confirmed detection of positive anti Toxocara IgG in $68.6 \%$ of adult asthmatics. Besides, the present study demonstrated a statistically significant relationship between Toxocara seropositivity and pet ownership, as well as the pathogenesis of wheezing in asthmatic adults' during the last 12 months. Toxocara seroprevalence has not been found associated with other variables among patients. No signifaicant correlation has been found between positive anti Toxocara IgG signs and high level of total IgE and eosinophilia concentration.

\section{Conflict of interest}

The authors declarethat they have no conflict of interest.

Aclnowledgement. This study was supported by University Malaya IPPP Research Grant (PG061-2014A).

\section{REFERENCES}

Aranzamendi, C., Sofronic-Milosavljevic, L. \& Pinelli, E. (2013). Helminths: immunoregulation and inflammatory diseases which side are Trichinella spp. and Toxocara spp. on? Journal of Parasitology Rresearch 2013: 329438. 
Buijs, J., Borsboom, G., Van Gemund, J.J., Hazebroek, A., Van Dongen, P.A., Van Knapen, F. \& Neijens, H.J. (1994). Toxocara seroprevalence in 5-year-old elementary schoolchildren: relation with allergic asthma. American Journal of Epidemiology 140(9): 839-847.

Buijs, J., Egbers, M.W. \& Nijkamp, F.P. (1995). Toxocara canis-induced airway eosinophilia and tracheal hyporeactivity in guinea pigs and mice. European Journal of Pharmacology: Environmental Toxicology and Pharmacology 293(3): 207-215.

Cobzaru, R.G., Rîpă, C., Leon, M.M., Luca, M.C., Ivan, A. \& Luca, M. (2012). Correlation between asthma and Toxocara canis infection. Revista medico-chirurgicala a Societatii de Medici si Naturalisti din Iassi 116(3): 727-30.

Doğan, N., Dinleyici, E.Ç., Bor, Ö., Töz, S.Ö. \& Özbel, Y. (2007). Seroepidemiological survey for Toxocara canis infection in the northwestern part of Turkey. Türkiye Parazitoloji Dergisi 31(4): 288-291.

Fan, C.K., Liao, C.W. \& Cheng, Y.C. (2013). Factors affecting disease manifestation of toxocariasis in humans: Genetics and environment. Veterinary Parasitology 193(4): 342-352.

Feldman, G.J. \& Parker, H.W. (1992). Visceral larva migrans associated with the hypereosinophilic syndrome and the onset of severe asthma. Annals of internal Medicine 116(10): 838-840.

Fernando, D., Wickramasinghe, P., Kapilananda, G., Dewasurendra, R.L., Amarasooriya, M. \& Dayaratne, A. (2009). Toxocara seropositivity in Sri Lankan children with asthma. $J$ Pediatrics Intermational 51(2): 241-245.

Hakim, S.L., Thadasavanth, M., Shamilah, R.H.R. \& Yogeswari, S. (1997). Prevalence of Toxocara canis antibody among children with bronchial asthma in Klang Hospital, MalaysiaJ Transactions of the Royal Society of Tropical Medicine and Hygiene 91(5): 528-528.
Kuk, S., Ozel, E., Oguzturk, H., Kirkil, G. \& Kaplan, M. (2006). Seroprevalence of Toxocara antibodies in patients with adult asthma. Southern Medical Journal 99(7): 719-722.

Kustimur, S., Dogruman Al, F., Oguzulgen, K., Bakir, H., Maral, I., Turktas, H. \& Tuzun, H. (2007). Toxocara seroprevalence in adults with bronchial asthma $J$ Transactions of the Royal Society of Tropical Medicine and Hygiene 101(3): 270-274.

Li, L., Gao, W., Yang, X., Wu, D., Bi, H., Zhang, S., Huang, M. \& Yao, X. (2014). Asthma and Toxocariasis. Annals of Allergy, Asthma \& Immunologol 113(2): 187192.

Ma, G., Holland, C.V., Wang, T., Hofmann, A., Fan, C.K., Maizels, R.M., Hotez, P.J. \& Gasser, R.B. (2018). Human Toxocariasis. Lancet Infectious Diseases 18(1): e14$\mathrm{e} 24$.

Mendonça, L.R., Figueiredo, C.A., Esquivel, R., Fiaccone, R.L., Pontes-de-Carvalho, L., Cooper, P., Barreto, M.L. \& AlcantaraNeves, N.M. (2013). Seroprevalence and risk factors for Toxocara infection in children from an urban large setting in Northeast Brazil J Acta Tropica 128(1): 90-95.

Mendonça, L.R., Veiga, R.V., Dattoli, V.C.C., Figueiredo, C.A., Fiaccone, R., Santos, J., Cruz, Á.A., Rodrigues, L.C., Cooper, P.J., Pontes-De-Carvalho, L.C., Barreto, M.L. \& Alcantara-Neves, N.M. (2012). Toxocara seropositivity, atopy and wheezing in children living in poor neighbourhoods in urban Latin American $J$ PLoS Neglected Tropical Diseases 6(1): e1886.

Mosayebi, M., Moini, L., Hajihossein, R., Didehdar, M. \& Eslamirad, Z. (2016). Detection of specific antibody reactivity to Toxocara larval excretory-secretory antigens in asthmatic patients (5-15 years). The Open Microbiology Journal 10: 162-167.

Pinelli, E. \& Aranzamendi, C. (2012). Toxocara infection and its association with allergic manifestations. Endocrine, Metabolic \& Immune Disorders - Drug Targets 12(1): 33-44. 
Qualizza, R., Incorvaia, C., Grande, R., Makri, E. \& Allegra, L. (2011). Seroprevalence of IgG anti-Toxocara species antibodies in a population of patients with suspected allergy. International Journal of General Medicine 4: 783-787.

Qualizza, R., Megali, R. \& Incorvaia, C. (2009). Toxocariasis resulting in seeming allergy. Iranian Journal of Allergy, Asthma and Immunology 8(3): 161-164.

Sharghi, N., Schantz, P.M., Caramico, L., Ballas, K., Teague, B.A. \& Hotez, P.J. (2001). Environmental exposure to Toxocara as a possible risk factor for asthma: a clinic-based case-control study. Clinical Infectious Diseases 32(7): e111-e116.
Silva, M.B., Amor, A.L.M., Santos, L.N., Galvão, A.A., Vera, A.V.O., Silva, E.S., Barbosa, C.G., Goncalves, M.S., Cooper, P.J., Figueiredo, C.A., Ribeiro, R.C. \& Neves, N.M.A. (2017). Risk factors for Toxocara spp. seroprevalence and its association with atopy and asthma phenotypes in school-age children in a small town and semi-rural areas of Northeast Brazil $J$ Acta Tropica 174: 158-164.

Yoshida, A., Hombu, A., Wang, Z. \& Maruyama, H. (2016). Larva migrans syndrome caused by Toxocara and Ascaris roundworm infections in Japanese patients. European Journal of Clinical Microbiology Infectious Diseases 35: 1521-1529. 